\title{
Ultrasonic fatigue testing under multiaxial loading conditions on a railway wheel
}

\author{
Pedro R. da $\operatorname{Costa}^{1}$, Henrique Soares ${ }^{1}$, Luís Reis ${ }^{1,2}$, and Manuel Freitas ${ }^{2,3}$ \\ ${ }^{1}$ Instituto Superior Técnico, Universidade de Lisboa, Av. Rovisco Pais, 1049-001 Lisbon, Portugal \\ ${ }^{2}$ IDMEC, Instituto Superior Técnico, Av. Rovisco Pais, 1049-001 Lisbon, Portugal \\ ${ }^{3}$ Atlântica, University School, Fabrica da Pólvora, Barcarena, 2730-036 Barcarena, Portugal
}

\begin{abstract}
Ultrasonic fatigue testing is a relative recent fatigue methodology that uses resonant principles for the induction of stress cycles in a specific designed material specimen. This experimental method can apply very high cycle frequency, the most common frequency being $20 \mathrm{kHz}$, and was created with the main purpose of studying material fatigue life in the Very High Cycle Fatigue regime between $10^{7}$ and $10^{9}$ cycles with a higher performance of time and energy wise in comparison to conventional servo-hydraulic machines. In this study an improvement of an already built multiaxial ultrasonic fatigue machine in the Instituto Superior Técnico laboratories was carried out to specific designed specimens and afterwards a fatigue study was made for a material of a worn-out railway wheel. The particular design of the specimen was achieved by numerical and experimental analysis based on previous experiments and components. Thermographic imaging and the application of rosette strain gauges to the main throat of the specimens were conducted in order to validate the improved specimen design and to understand the real induced stresses on the specimen. Afterwards fatigue tests were conducted for several specimens for a wide range of stresses with a stress ratio $\mathrm{R}=-1$ and an axial vs shear stress ratio of around 0.58 . Results were analysed and fracture analysis was also carried out.
\end{abstract}

\section{Introduction}

The reliability of any given structure or machine subjected to dynamic loads is mainly dependent on the detailed fatigue study of the applied materials. As the demand for higher life cycles in applications subjected to complex stresses increases, so does the necessity for new and more multifaceted fatigue testing methods. Since it was proven before that a fatigue endurance limit should no longer be considered [1], ultrasonic fatigue tests were developed for the study of the life beyond that point. This is now known as the Very High Cycle Fatigue (VHCF) regime. In these tests, specimens are subjected to alternating stresses with frequencies as high as $20 \mathrm{kHz}$.

Ultrasonic fatigue tests today can apply uniaxial stresses as in $[2,3]$ for tension/compression, [4] pure torsion and [5] bending, but it is important to be able to apply

\footnotetext{
Corresponding author: luis.g.reis@tecnico.ulisboa.pt
} 
complex multiaxial loads since most real components are subjected to a complex stress state when under cyclic loading. Very few examples of multiaxial ultrasonic fatigue testing can be found, one worthy mention is the test created by Palin-Luc able to achieve biaxial bending stresses [6]. In this study the fatigue analysis of a worn out railway wheel is performed, focuses on the development and testing of an ongoing research of a multiaxial tension/torsion ultrasonic fatigue machine $[7,8,9]$.

\section{Material and Methodology}

\subsection{Material}

The material under study is a horn out railway wheel and all the specimens were directly machined from the its rim, as presented in figure 1. For the ultrasonic fatigue tests some properties are required for specimen design. Such properties were taken through mechanical tests and are presented in Table 1.

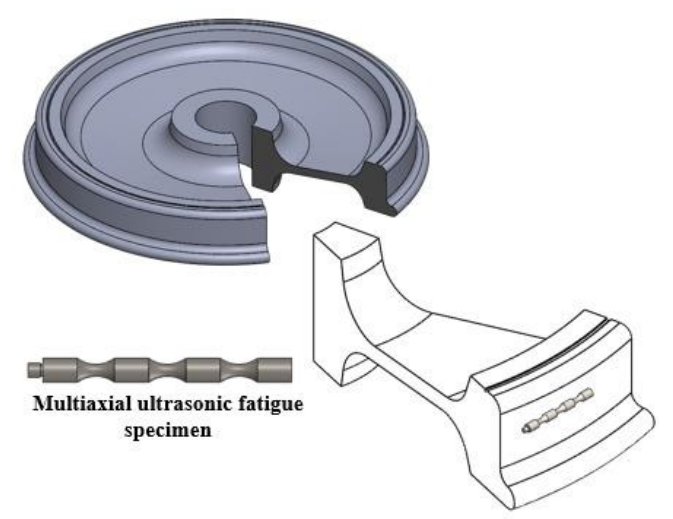

Fig. 1. Railway wheel representation and machined location and orientation of the specimens

Table 1. Mechanical properties of the railway wheel in study at the rim region

\begin{tabular}{|c|c|c|}
\hline Young Modulus, E $(\mathrm{GPa})$ & Density, $\rho\left(\mathrm{kg} / \mathrm{m}^{3}\right)$ & Hardness Vickers $(\mathrm{HV})$ \\
\hline $201 \mathrm{GPa}$ & 7.85 & {$[270-300]$} \\
\hline
\end{tabular}

\subsection{Multiaxial ultrasonic fatigue testing}

When designing an ultrasonic fatigue test, the resonance of all the attached components is a needful of consideration for any kind of applying stress of interest. Within the transducer's frequency, the resonant mode of each component will create the composed resonant mode when all are attached, being important that they have displacement agreement and similar frequencies.

The ultrasonic fatigue machine used in this study applies a biaxial state of stress, inducing cyclic axial tension/compression and torsion stresses in phase. This fatigue testing device is being developed in the Instituto Superior Técnico laboratories [8]. The test follows the basis of the uniaxial tension/compression test and alters the horn and specimen geometry for the induction of torsion stresses.

As described by the research [9], the multiaxiality is achieved by exciting the specimen not in one but two resonance modes, the first axial and the third rotational mode. This is possible by the special horn geometry capable of transforming part of the axial displacements in rotation, thus transmitting simultaneously axial and rotational excitation to the specimen. 
A simple representation of the horn and specimen with a few specimen designations is presented in figure 2 .

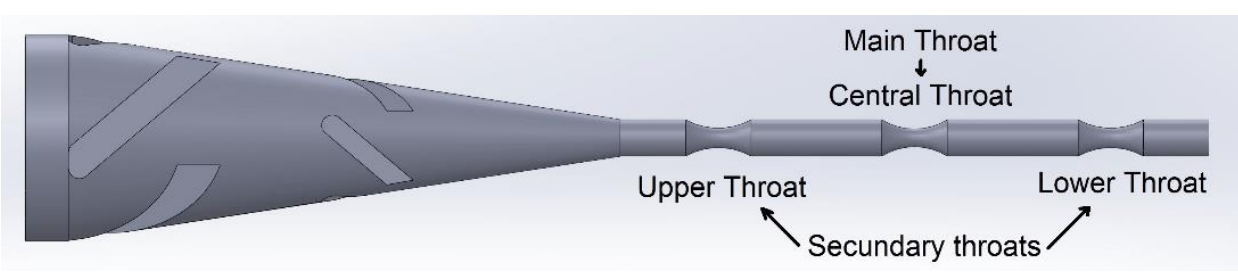

Fig. 2. Representation of the Horn and Specimen with specimen's key designations

Throughout the development and study of this multiaxial ultrasonic fatigue machine, several alterations and improvements have been made in the interest of a simpler, more stable and credible testing device. New horns have been machined and an improved specimen geometry was created. This new and improved horn and specimens' geometries have been employed and tested in this study. A representation of both resonant modes displacements (in regard the highest displacement direction, Uy for axial, Ur for torsion) as well as the resonant mode combination magnitude displacement (Umag) is presented in Figure 3. They also provide an important key tool for the multiaxial relation axial vs shear stress study.

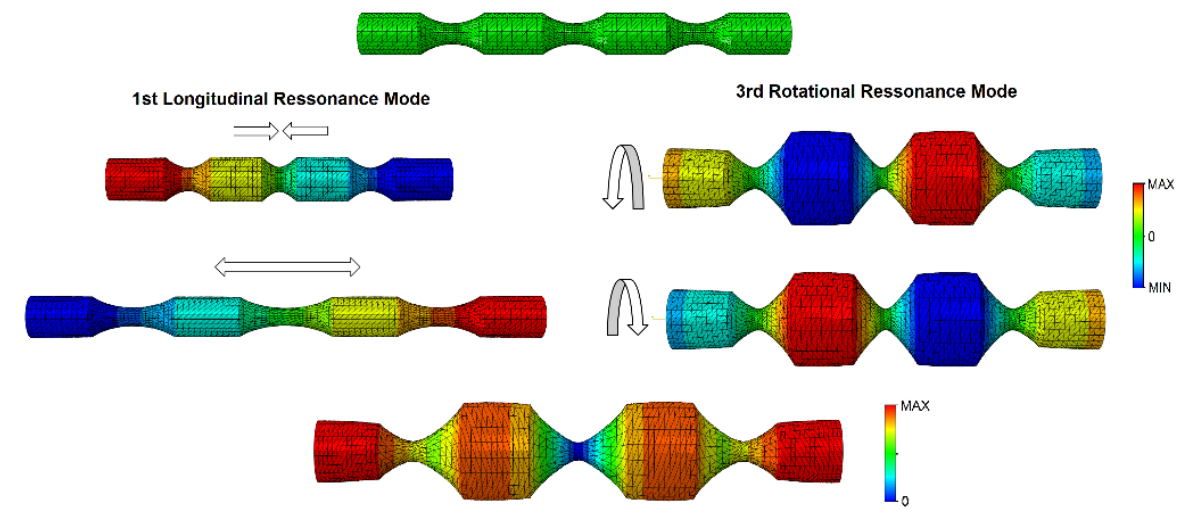

Fig. 3. Representation of the displacement of the specimen's resonant modes of interest when excited individually and simultaneously

\subsection{Methodology}

Following the initial specimen idea described in [9] with new relations between dimensions, several combinations are tested in a finite element software until one final combination has both the mentioned resonant modes, figure 2, with similar frequency and close as possible to the middle range of the transducer frequency of $20 \mathrm{kHz}$.

After machining the specimen in accordance, they are black painted for temperature measurement, being with a thermal camera or with a pyrometer. Before applying a strain gauge, the specimens were first tested to see if resonance was possible to achieve and if the highest heat generation was within the main throat. Afterwards a rosette strain gauge is applied to the main throat and several readings for several power settings were made following the same methodology as in the research [10]. With the results a calibration, 
power/applied stress was taken, and the remaining specimens are subsequently tested to failure.

All tests are conducted with a temperature control, meaning that the transducer will only work until the specimen reaches a certain temperature and will be on stand by until it cools for a determined lower temperature. All the conducted tests had an established temperature range between $27^{\circ}-50^{\circ}$ degrees.

Along a test when a fatigue crack is nucleated the specimen losses stiffness and therefore resonance for the working frequency. Thus, the test comes to a stop and the specimen is withdrawn from the ultrasonic fatigue machine. The specimen is afterwards placed in a tensile machine for full failure, making the fatigue fracture visible for its analysis.

\section{Results}

As mentioned, the specimens were analysed using the transducer frequency scan to see if resonance was possible. Simultaneously a thermal camera was placed, and several pictures were taken for several specimens, proving the higher stress combination is being induced at the central throat, as is showed in figure 4.

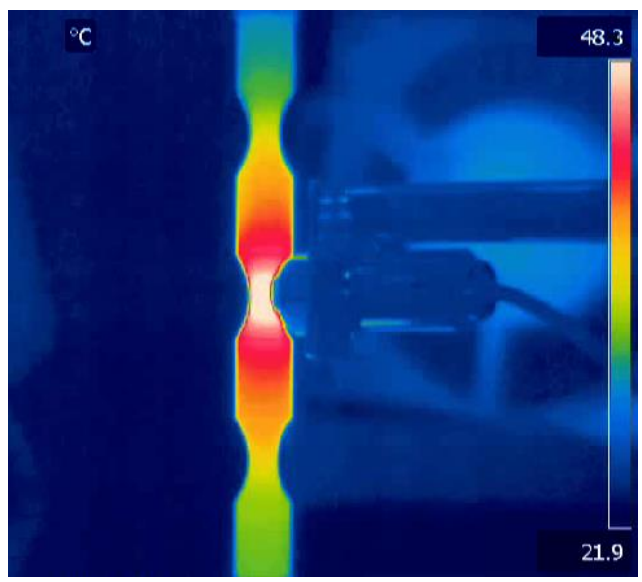

Fig. 4. Thermographic analysis while testing

Confirming a possible resonance and higher stress combination on the main throat, a roseate strain gauge was subsequently placed on one specimen for the strain measurement. The stress calculation results made from the measured strain are presented in figure 5 and show the existence of the axial stress (Oy), shear stress created by the torsion (Oxy), and from it the axial vs shear stress ratio $\left(\sigma_{r}=\sigma_{x y} / \sigma_{y}\right)$ can be calculated, being around 0.58 after an initial lower values to lower power settings. 


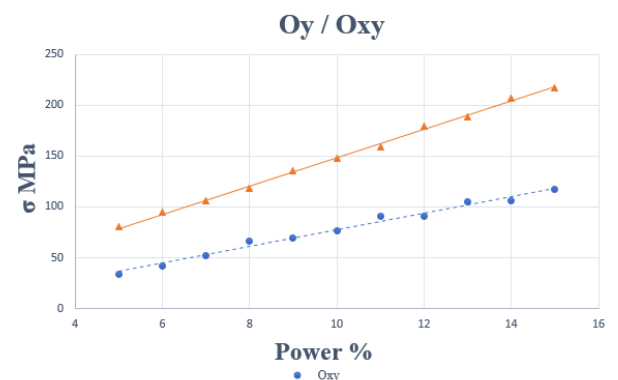

(A)

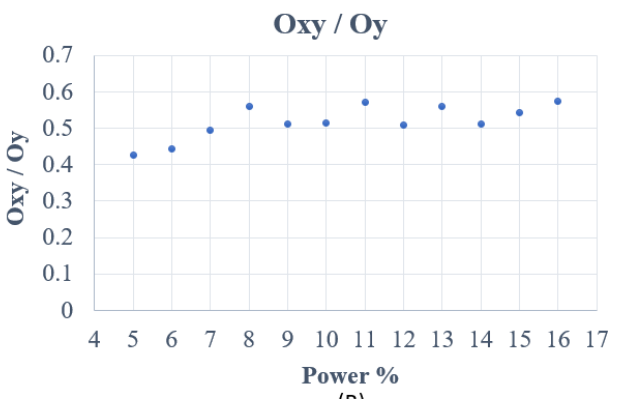

(B)

Fig. 5. Stress power calibration results from the strain measurements: (A) Axial Oy and Shear Oxy stress; (B) Stress shear/axial relation

Afterwards several stress values were tested by applying different power settings. All the tested specimens reached to failure, being with the first stress combination applied or after reaching $10^{9}$ cycles being yet again under test but with a higher stress combination. Figure 6 presents the S-N results.

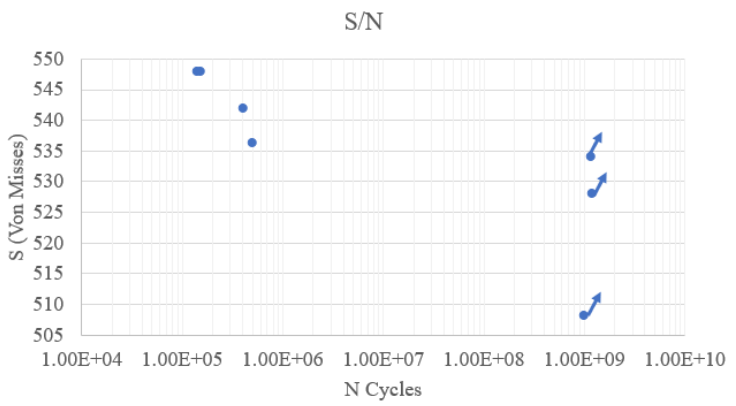

Fig. 6. S-N results

Looking at the entire fracture surface, see Fig. 7, the crack initiation point was always located at the specimen's surface. It should also be mentioned that the percentage of fatigue area is much lesser in comparison to uniaxial fatigue specimens obtained by the research [7].

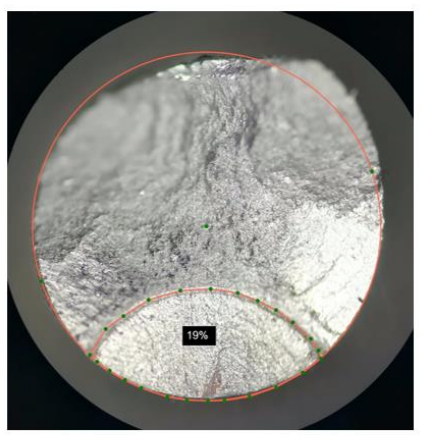

(A)

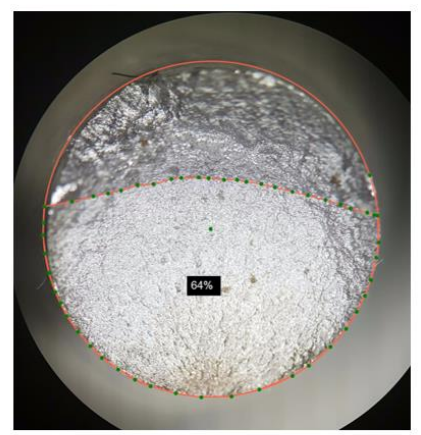

(B)

Fig. 7. Fatigue fracture surface: (A) Multiaxial specimen with 19\%; (B) Uniaxial specimen with $64 \%$ 


\section{Conclusions}

The new specimen geometry proved to have better stability than the previous geometries by having all the tested specimen's capability of being excited in resonance and by having the highest stress combination on the main throat. Now a less of a high-resolution machining is required for a specimen capable of being excited simultaneously in two resonant modes correctly. Thus, the multiaxial stress was achieved and proved by the strain gauges measurements.

It was also possible to reach failure to all the tested specimens with a clear fatigue fracture surface. The lower percentage in fatigue surface area is related to the excitation of the specimen being related with not one but two simultaneous resonant modes that makes it be more sensible to the loss of integrity, thus, it losses the capability of resonance at the transducer range of work much quicker than a uniaxial specimen.

The stress life results showed to have a correlation for the higher stresses vs lower life cycles. Still many of which reached $10^{9}$ cycles without breaking.

The authors fully acknowledge the support and financing provided by FCT for the Multiaxial Very High Cycle Fatigue project PTDC/EMS-PRO/5760/2014

This work was also supported by FCT, through IDMEC, under LAETA, project UID/EMS/50022/2019. and also supported by Progama Ciência sem Fronteiras, project 9999.013107/2013-05.

\section{References}

[1] C. Bathias and P. C. Paris, Gigacycle fatigue in mechanical practice. 2005.

[2] R. Pérez-Mora, T. Palin-Luc, C. Bathias, and P. C. Paris, Very high cycle fatigue of a high strength steel under sea water corrosion: A strong corrosion and mechanical damage coupling, Int. J. Fatigue, 74, 156-165, (2015).

[3] A. C. Grigorescu, P. M. Hilgendorff, M. Zimmermann, C. P. Fritzen, and H. J. Christ, Cyclic deformation behavior of austenitic $\mathrm{Cr}-\mathrm{Ni}$-steels in the VHCF regime: Part I Experimental study, Int. J. Fatigue, 93, 250-260, (2016).

[4] A. Nikitin, C. Bathias, and T. Palin-Luc, A new piezoelectric fatigue testing machine in pure torsion for ultrasonic gigacycle fatigue tests: Application to forged and extruded titanium alloys, Fatigue Fract. Eng. Mater. Struct., 38, 11, 1294-1304, (2015).

[5] H. Q. Xue, H. Tao, F. Montembault, Q. Y. Wang, and C. Bathias, Development of a three-point bending fatigue testing methodology at $20 \mathrm{kHz}$ frequency, Int. J. Fatigue, 29, 2085-2093, (2007).

[6] C. Brugger, T. Palin-Luc, P. Osmond, and M. Blanc, Gigacycle fatigue behavior of a cast aluminum alloy under biaxial bending: experiments with a new piezoelectric fatigue testing device, 21st Eur. Conf. Fract. ECF21, 2, 96-100, (2016).

[7] H. Soares, P. Costa, M. Freitas, and L. Reis, Fatigue life assessment of a railway wheel material under HCF and VHCF conditions, MATEC Web Conf., 165, 09003, (2018).

[8] M. Vieira, M. De Freitas, L. Reis, A. M. R. Ribeiro, and M. Da Fonte, Development of a very high cycle fatigue (VHCF) multiaxial testing device, Frat. ed Integrita Strutt., 10, 37, 131-137, (2016).

[9] P. Costa, M. Vieira, L. Reis, A. Ribeiro, and M. de Freitas, New specimen and horn 
design for combined tension and torsion ultrasonic fatigue testing in the very high cycle fatigue regime, Int. J. Fatigue, 103, 248-257, (2017).

[10] M. Vieira, L. Reis, M. Freitas, and A. Ribeiro, Strain measurements on specimens subjected to biaxial ultrasonic fatigue testing, Theor. Appl. Fract. Mech., 85, 2-8, (2016). 